\title{
Role of Adipose-Derived Mesenchymal Stem Cells in Full Thickness Wound Healing
}

Iswinarno Dososaputro', Lisette T Hoekstra ${ }^{2}$, Dinar Rahmania ${ }^{1}$ and David S Perdanakusuma

iPlastic Surgery Department of Airlangga University School of Medicine / Dr. Soetomo General Hospital, Surabaya, Indonesia

${ }^{2}$ Department of Plastic, Reconstructive, and Hand surgery, Maastricht University Medical Centre, The Netherlands

\begin{abstract}
Background: Wound healing problems can arise in donor wounds after harvesting a full-thickness graft. Returning Mesenchymal Stem Cells (MSCs) on the wounds may accelerate wound healing. The aim of this study is to analyse the effect of MSCs in the epithelialization process and collagen density on full-thickness wound healing.

Methods: The pilot study included 10 patients undergoing excision of a full-thickness skin graft on the groin. Patients were randomly divided into two groups: Mesenchymal Stem Cells (MSCs) and Non-Mesenchymal Stem Cells (Non-MSCs). The MSCs group had previously undergone fat harvesting which was processed into mesenchymal stem cells. Biopsies were taken from both groups on days 14 (proliferative phase) and 45 (maturation phase), and were compared with normal skin (NS; $n=5$ ). Epithelial layers of the epidermis were assessed with hematoxylin eosin staining. Collagen density was evaluated with MT staining, and analysed using a light microscope.

Result: In the MSCs group and the Non-MSCs group, the number of epithelial layers were significantly higher compared to the NS-group on day $45(14.7 \pm 0.70$ and $8.24 \pm 0.76$ vs $5.43 \pm 0.60$ respectively; $p<0.001$ and $p<0.001)$. The collagen density in the MSCs group on day 14 was $33.3 \pm 2.46 \%$ in the MSCs group and $45.7 \pm 5.84 \%$ in the non-MSCs group, compared to $54.3 \pm 3.71 \%$ in the NS-group ( $p<0.001$ and $p=0.012$ resp.). These values increased on day 45 to $49.2 \pm 3.28 \%$ in the MSCs group, and $73.4 \pm 1.63 \%$ in the non-MSCs group.

Conclusion: Mesenchymal stem cells increased the number of epithelial layers in the full-thickness wound healing process compared to normal skin. A higher increase was seen in the MSCs-group. On day 45, an increase in collagen density was observed in the MSCs group and Non-MSCs group. Adipose-derived mesenchymal stem cells can be used in the process of full-thickness wound healing. Future randomized controlled trials are needed to confirm these findings.
\end{abstract}

Keywords: Mesenchymal stem cells, Non-Mesenchymal Stem Cells, Collagen Density, Epithelial layer, Adipose-derived stem cells, Fullthickness graft

\section{Background}

Full-Thickness Skin Grafts (FTGs) are often used in reconstructive burn surgery, because they provide a better aesthetic result and less contracture compared to Split Thickness Skin Grafts (SSGs) [1]. However, full-thickness donor wounds can cause problems in wound healing or during closure. The groin area is frequently used as donor site of FTG, although it is an area of motion. This can result in extensive tension on the wound edges which causes dehiscence or necrosis. The final result of the full-thickness wound is a scar, with sometimes the formation of a hypertrophic scar.

It is well known that human Mesenchymal Stem Cells (MSCs) can accelerate the wound healing process by their self-renewing ability. Mesenchymal stem cells have been used in several studies, which showed that regeneration of full-thickness wounds can be enhanced by application of MSCs [1-4]. For example, Bone Marrow derived Mesenchymal Stem Cells (bMSCs) have the potential to differentiate into osteoblasts, chondrocytes, myocytes [5-8], and adipocytes [2,5,7].

Fu et al. reported that bMSCs cells improved the quality of wound healing [3]. Furthermore, in vivo and animal grafting experiments with MSCs showed that several aspects of wound regeneration were increased, such as the reepithelialization process, the regeneration of the epidermis, and the amount of microvasculature, fibroblasts and collagen. The conclusion of this study was that perfect skin regeneration was achieved with MSCs after full-thickness wounds [3]. Also, the outcomes of Deng et al. were promising, with the application of MSCs in mice transplanted into other irradiated mice [2]. They reported that bMSCs can contribute to skin functional cells and regeneration.

Mesenchymal Stem Cells Derived from Adipose Tissue (ADSCs) can also increase the wound healing process and inhibit hyperplastic scar formation by down-regulation of TGF- $\beta 1$, collagen type I and III [9]. Some advantages have been described for ADSCs compared to bMSCs, such as the relatively easy harvesting of a large amounts of adipose tissue, less pain during harvesting, and the multi-lineage capacity of differentiating into several cell types. Lo Furno et al. described the potential therapeutic applications of these cells which can differentiate into adipogenic, osteogenic, chondrogenic, and neurogenic cells. Besides that, they also reported the possibility of differentiating into muscle, epithelial, pancreatic, and hepatic cells [10].

There is a high interest in using stem cells for wound healing, since they can migrate to the wound site, and become part of the microenvironment. Besides that, stem cells can increase growth factors [11],

*Corresponding author: Lisette $T$ Hoekstra, Department of Plastic, Reconstructive, and Hand surgery, Maastricht University Medical Centre The Netherlands, Tel: +31 433877548; E-mail: It_hoekstra@hotmail.com

Received August 05, 2017; Accepted August 28, 2017; Published September 05, 2017

Citation: Dososaputro I, Hoekstra LT, Rahmania D, Perdanakusuma DS (2017) Role of Adipose-Derived Mesenchymal Stem Cells in Full Thickness Wound Healing. J Stem Cell Res Ther 7: 398. doi: 10.4172/2157-7633.1000398

Copyright: (c) 2017 Dososaputro I, et al. This is an open-access article distributed under the terms of the Creative Commons Attribution License, which permits unrestricted use, distribution, and reproduction in any medium, provided the original author and source are credited. 
reduce inflammation, and inhibit hypertrophic scar formation $[3,11]$. MSCs accelerate the epithelialization process, and thus the wound closure. Furthermore, MSCs improve the quality of tissue tension, help chronic wound healing and minimize scarring. Therefore, mesenchymal stem cells are expected to become helpful in accelerating healing of full-thickness wounds. However, the underlying mechanism is still not fully understood. Therefore, the aim of this study is to analyse the effect of MSCs in the epithelialization process and collagen density on fullthickness wound healing.

\section{Materials and Methods}

Ten patients undergoing surgical excision of a FTG on the groin were included in this pilot study. The full-thickness graft wound was primary closed. These patients were randomly divided into two groups: Mesenchymal Stem Cells (MSCs; $n=5$ ) and Non-Mesenchymal Stem Cells or control group (Non-MSCs; $n=5$ ). The MSCs group had previously undergone surgical removal of abdominal fat blocks of 2 $\times 2 \mathrm{~cm}$ size which were processed into mesenchymal stem cells in the laboratory of the Dr. Soetomo Hospital Tissue Bank for 14 days. These mesenchymal stem cells were administered intra-dermally to the wound in the groin in the MSCs group. Biopsies were taken from both groups on days 14 (proliferative phase) and day 45 (maturation phase). These outcomes were compared with normal skin biopsies (NS; $n=5$ ). NS biopsies were prepared from the Anatomical Pathology Laboratory of the University of Airlangga, Surabaya. The assessment and comparison of the epithelial layers of the epidermis were performed with haematoxylin eosin staining. Collagen density was evaluated with Masson trichrome staining and then analysed using a light microscope type CX 21. Photos were made on day 14 and 45 to compare visual outcomes. A flowchart of the research protocol is shown in Figure 1. There were no patients with diabetes or obesity. This study was approved by the institutional review board of the University of Airlangga, Surabaya.

\section{Statistical Analysis}

Data are tested for normal distribution and equal variances. The values are expressed as means with standard deviation, unless otherwise stated. Data were analysed using one-way ANOVA. Statistical analysis was accepted when $\mathrm{p}<0.05$. The data were analysed by statistical software (SPSS 22.0; SPSS; Chicago; IL).

\section{Results}

\section{Clinical appearance}

In the MSCs group, the wound surface slightly raised, no dehiscence was obtained, and the colour of the skin was comparable with the surrounding tissues on day 14. However, in the Non-MSCs group, surgical wound surface was irregular, dehiscence was seen, and hyperaemia was observed at the edge of the stitches on day 14. On day 45 , the MSCs group showed that the surface of the scar was at the same level with the surrounding tissues, and the colour was more pale. In the Non-MSCs group, there was scarring along the stitches, with a mean scar width of 3-4 mm. Besides that, the surrounding skin was darker and more elevated (Figure 2).

\section{Epithelial layers}

The mean number of epithelial layers on day 14 in the MSCs group was $10.28 \pm 1.36$, compared to $8.50 \pm 1.19$ in the Non-MSCs group ( $\mathrm{p}=0.031$ ). In the NS-group, the number of epithelial layers was only $5.43 \pm 0.60$. This was significantly different from the MSC group $(\mathrm{p}<0.001)$, and the non-MSCs group $(\mathrm{p}=0.002)$. On day 45 , the mean number of epithelial layers in the MSCs group was $14.7 \pm 0.70$, thus increased double compared to the number of layers of the epithelium in the group of NS $(5.43 \pm 0.60)$. For the Non-MSCs group, this number was $8.24 \pm 0.76$ on day $45,1.5$ times higher than the NS-group. The outcomes further show that the number of epithelial layers for the MSCs

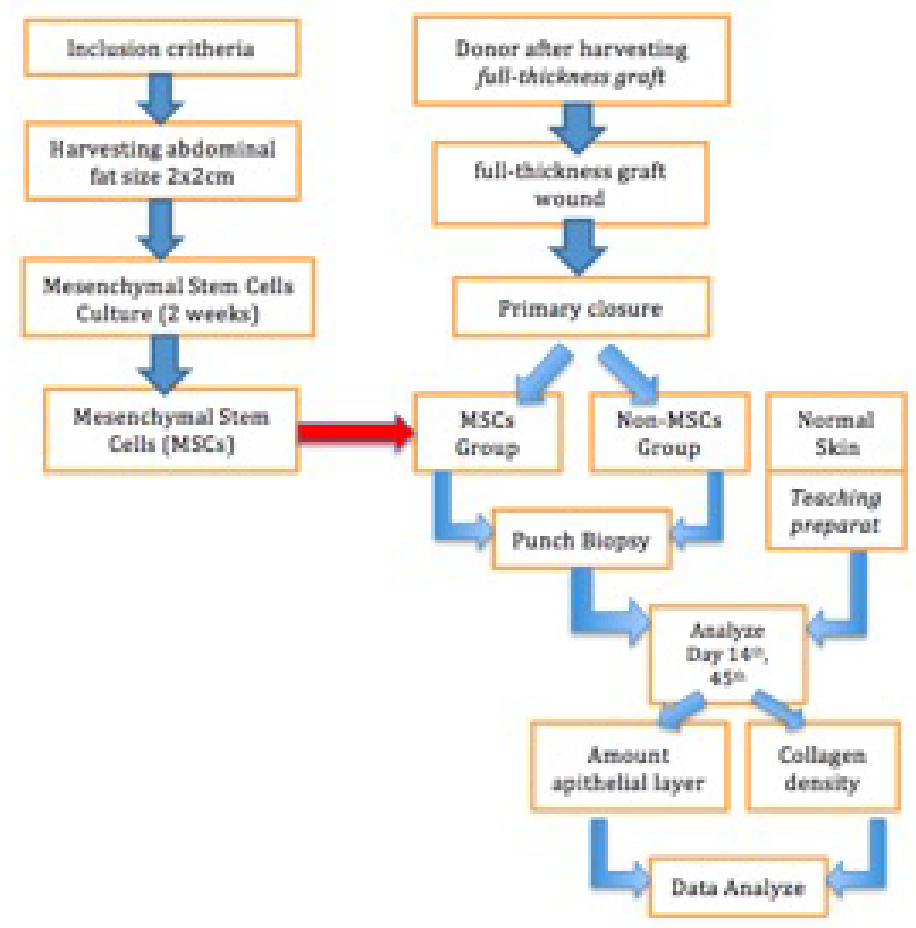

Figure 1: A flowchart of the research protocol. 
Citation: Dososaputro I, Hoekstra LT, Rahmania D, Perdanakusuma DS (2017) Role of Adipose-Derived Mesenchymal Stem Cells in Full Thickness Wound Healing. J Stem Cell Res Ther 7: 398. doi: 10.4172/2157-7633.1000398

Page 3 of 6

and Non-MSCs group on day 14 and 45 are significantly higher than the NS-group $(\mathrm{p}<0.001)$. The results are described in Table 1. Histological appearance with HE staining of epithelial layers on NS-group compared to the Non-MSCs group and MSCs group are shown in Figure 3.

\section{Collagen density}

On day 14 , collagen density was $33.3 \pm 2.46 \%$ in the MSCs group.
For the Non-MSCs group, collagen density was $45.7 \pm 5.84 \%$ on day 14 , which is both lower than the collagen density in the NS-group (54.3 \pm $3.71 \%)$. On day 45 , the collagen density increased to $49.2 \pm 3.28 \%$ in the MSCs group, and to $73.4 \pm 1.63 \%$ in the non-MSC group. Both groups were significantly different from the NS-group $(54.3 \pm 3.71 \%$; $\mathrm{p}=0.026$ and $\mathrm{p}<0.001$ respectively). Results are shown in Table 2 and Figure 4.

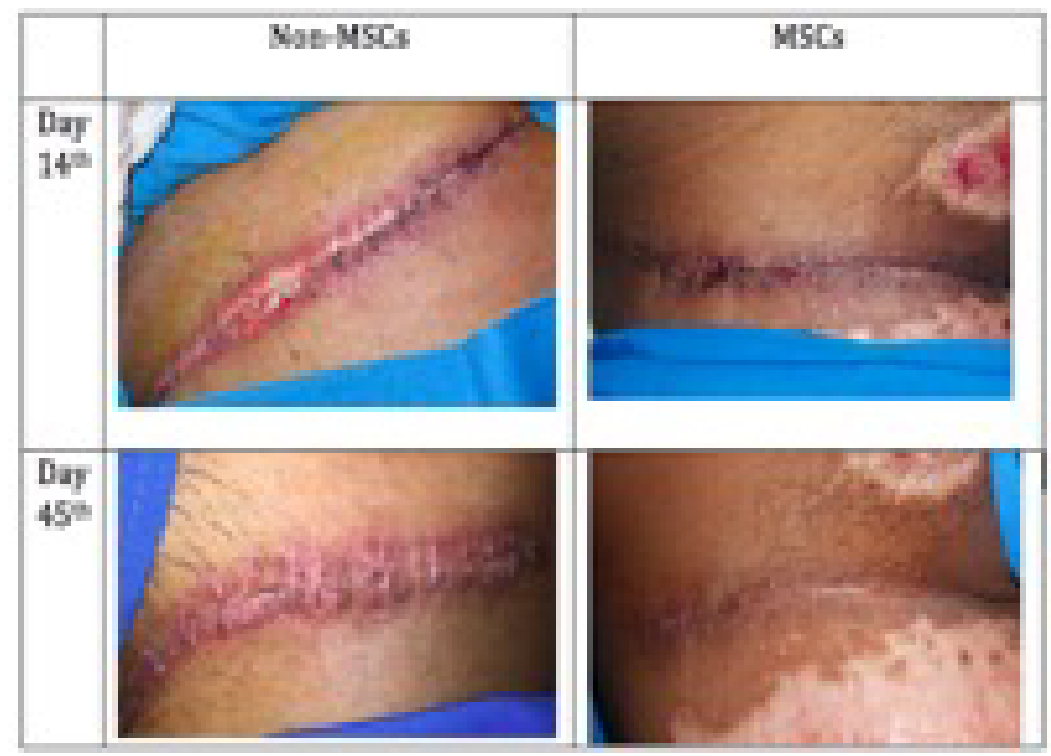

Figure 2: Histological appearance of Non-MSCs group and MSCs group.

\begin{tabular}{|c|c|c|c|c|c|}
\hline Amount Epithelial Layers & Normal Skin & MSCs & Non-MSCs & P-value (NS vs MSCs) & P-value (NS vs Non-MSCs) \\
\hline Day 14 & $5.43 \pm 0.60$ & $10.28 \pm 1.36$ & $8.50 \pm 1.19$ & $<0.001$ & 0.002 \\
\hline Day 45 & $5.43 \pm 0.60$ & $14.7 \pm 0.70$ & $8.24 \pm 0.76$ & $<0.001$ & $<0.001$ \\
\hline
\end{tabular}

Table 1: Results of HE staining of epithelial layers on NS-group compared to the Non-MSCs group and MSCs group.

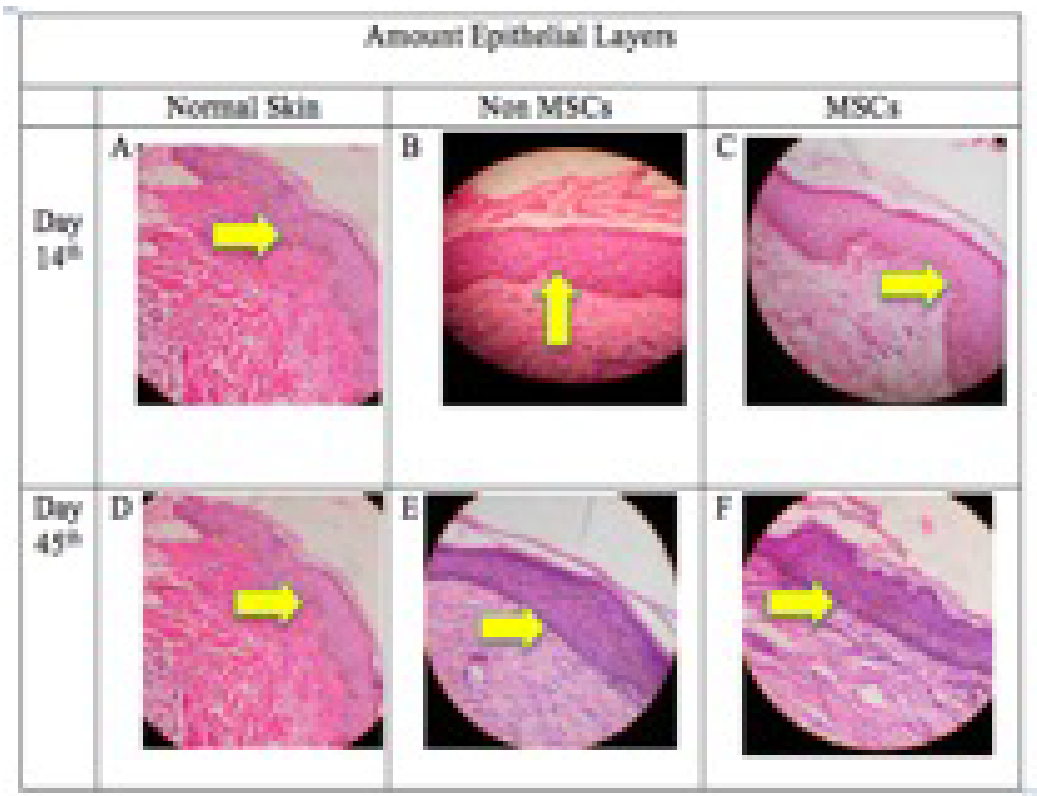

Figure 3: Histological appearance with HE staining of epithelial layers on NS-group compared to the Non-MSCs group and MSCs group. 
Citation: Dososaputro I, Hoekstra LT, Rahmania D, Perdanakusuma DS (2017) Role of Adipose-Derived Mesenchymal Stem Cells in Full Thickness Wound Healing. J Stem Cell Res Ther 7: 398. doi: 10.4172/2157-7633.1000398

Page 4 of 6

\begin{tabular}{|c|c|c|c|c|c|}
\hline Amount Epithelial Layers & Normal Skin & MSCs & Non-MSCst & P-value (NS vs MSCs) & P-value (NS vs Non-MSCs) \\
\hline Day 14 & $5.43 \pm 0.60$ & $10.28 \pm 1.36$ & $8.50 \pm 1.19$ & $<0.001$ \\
\hline Day 45 & $5.43 \pm 0.60$ & $14.7 \pm 0.70$ & $8.24 \pm 0.76$ & $<.002$ \\
\hline
\end{tabular}

Table 2: Results of collagen density on NS-group compared to the Non-MSCs group and MSCs group.

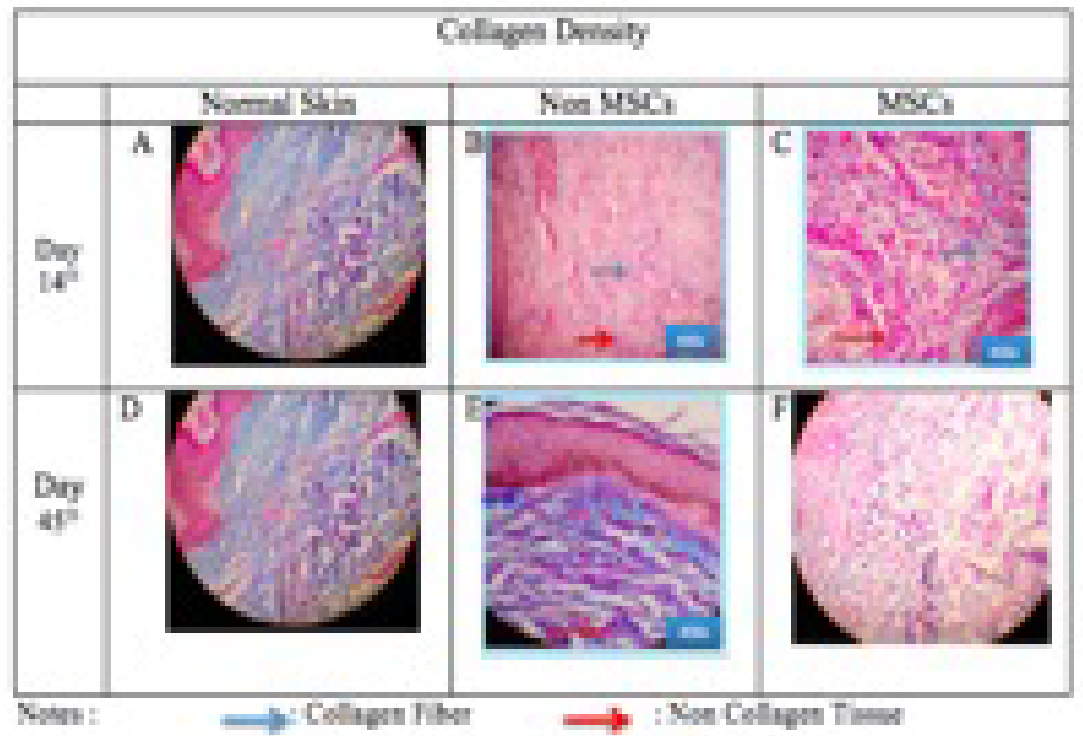

Figure 4: Histological appearance with Collagen Density on NS-group compared to the Non-MSCs group and MSCs group.

\section{Discussion}

Wounds that exist after harvesting a full thickness graft in the donor area can cause wound healing problems. This results in bad scars, which can even become hypertrophic in patients with a high Fitzpatrick scale, as mainly seen in Indonesian patients. This will lead to less aesthetic outcomes.

Some studies reported the effect of mesenchymal stem cells in wound healing, and demonstrated that mesenchymal stem cells stimulate re-epithelialization, suppress the inflammatory process, and has an anti-scarring effect [11-14]. However, the mechanism involved is still not elucidated. Several important factors are responsible for the alteration in wound healing function. Multifactorial processes take place in the proliferation phase of wound healing, such as angiogenesis, epithelialization, collagen deposition, and wound contraction. Chen et al. observed the epithelialization time of wounds and the hyperplastic scar formation time in New Zealand rabbits in which a ADSCs group was compared with a control group ( $n=36$ per group) [9]. ADSCs were injected in a wound on the left ear, while the wound on the right ear was injected with phosphate buffer solution. In the ADSCs group, hyperplastic scars were smaller, more flat, and soft. Epithelial cell layers were increased in comparison to the control group, a similar finding as in our study. However, they reported that the collagen density of hyperplastic scars was significantly decreased and arranged regularly in the ADSCs group. We could not confirm this result in our preliminary study. Furthermore, they showed a lower collage type I and III, and TGF- $\beta 1$ in the ADSCs group [9]. Lee et al. reported that the proliferation of ADSCs were increased by hypoxia, and improved the woundhealing function by secretion of Vascular Endothelial Growth Factor (VEGF) and basic fibroblast growth factor (bFGF) [11]. VEGF induces angiogenesis and fibroblasts are necessary to close the wound $[15,16]$. Also Jackson et al. described the role of VEGF and FGF in proliferation, migration and differentiation of microvascular endothelial cells [12]. Excessive fibrosis is seen in hypertrophic scars and is caused by an unusual proliferation of fibroblasts [17]. Keratinocytes in hypertrophic scars instruct fibroblasts to produce growth factors as Keratinocyte Growth Factor (KGF)/Fibroblast Growth Factor-7 (FGF7), interleukin-6 (IL-6), Hepatocyte Growth Factor (HGF) and (GM-CSF) which follow the same paracrine pattern [18-21]. Paracrine activities are important for wound healing. Some reports described the paracrine role of MSCs in wound healing $[22,23]$. Stem cells can be obtained easily from fat tissue, better than stem cells harvested from bone marrow.

In this preliminary study, administration of mesenchymal stem cells in the donor wound area, after excision of a full-thickness graft, can regulate the number of layers of epithelial. In the group of MSCs and Non-MSCs significantly differences were found in number of epithelial layers compared to the Normal Skin (NS). The number of epithelial layers in the MSCs group exceeded even two times the number of epithelial layers in the structure of the NS-group $(p=<0.001)$. A study of Sugiyama et al. also harvested mesenchymal stem cells from adipose tissue [24]. Their analyses revealed that several factors supported various epithelial cell markers (like p63, K15, K14) and thus cell growth, a similar finding as in our study.

This study showed that mesenchymal stem cells are capable of controlling the density of collagen in the maturation phase. On microscopic evaluation in the MSCs group, relatively stable collagen density was seen on day 14 (proliferative phase) with a density of $33.3 \%$ compared to the surrounding connective tissue. Progression of wound healing was observed in the dermis structure by administering 
mesenchymal stem cells in the MSCs group, which reached a density of $49.2 \%$ in the maturation phase, not far from the density of the dermis structure of the normal skin group (54.3\%). The density of collagen in the group of Non-MSCs seemed $45.7 \%$ on day 14 , however, more solid and irregular on day 45 , with rates up to $73.4 \%$. In the non-MSCs group collagen density exceeded the density of collagen in the NS-group. Maharlooei et al. evaluated the volume density of collagen fibres in a control group and treatment group in a diabetic rat model where a full-thickness wound was made on the dorsum of the rat $(n=26)$ [25]. No significant differences were found between both groups in volume density of collagen fibres, however, they showed a higher wound healing rate in rats with diabetes. Their conclusion was in accordance with our results, believing that adipose-derived mesenchymal stem cells promote the process of full-thickness wound healing.

Mesenchymal stem cells can help diminishing wound tension. In our pilot study, the donor wound in the groin area showed loose stitches on day 14 , however, there was no dehiscence. On day 45 , during the maturation phase, hypopigmentation was observed and scar colour was similar to the surrounding healthy skin.

Our study suffers from some limitations. The number of patients was small, so the results should be interpreted with caution. Larger prospective follow-up studies are crucial to determine if the epithelialization process and collagen density still increase in fullthickness wounds. Besides that, we only analysed the effect of MSCs in the epithelialization process and collagen density on full-thickness wound healing, although several other processes are described influencing the process of wound healing, such as angiogenesis, growth factors, and the inflammatory response.

Specific cell subpopulations of human MSCs have a stronger therapeutic efficacy than others, such as the vascular cell adhesion molecule-1 (VCAM-1+) and CD105+CD34- cells. VCAM-1, also known as CD106, is a cytokine regulated effector molecule which plays an important role in inflammation. This molecule is expressed by various types of cells, including mesenchymal cells. Du et al. isolated MSCs from placenta chorionic villi, and these were intramuscularly injected into a $\mathrm{BALB} / \mathrm{c}$ nude mice model of vascular ischemic disease [26]. This study showed that there was a functional improvement compared with mice that were not transplantated with VCAM-1+. Thus, VCAM-1+ cells exert angiogenic activities. Another study of Czapla et al. investigated the potential effect of MSCs with CD105+CD34- phenotype in a mouse model of limb ischaemia and myocardial infarction [27]. MSCs were isolated from human hearts and transplanted into mice. They concluded that 14 days post-injury the capillary density was increased. Besides that, faster wound healing was seen in ischemic muscles that were administered with MSCs [27]. These outcomes suggest that isolation of cell subpopulations of MSCs can have therapeutic clinical potential.

In conclusion, mesenchymal stem cells increased the structure of epithelial in wound healing process of full-thickness donor wounds during the observation period day 14th to day 45th in the MSC group in our study, compared to the non-MSCs group and NS group. The mesenchymal stem cells regulate the density of collagen in the proliferative phase and the initial phase of maturation in full-thickness wounds donor. Therefore, adipose-derived mesenchymal stem cells can be used in the process of full-thickness wound healing. However, randomized controlled trials with a larger group of patients are needed in the future to confirm these findings.

\section{References}

1. Harrison CA MacNeil S (2008) The mechanism of skin graft contraction: an update on current research and potential future therapies. Burns 34: 153-163. [PubMed]

2. Deng W, Han Q, Liao L, Li C, Ge W, et al. (2005) Engrafted bone marrowderived flk-(1+) mesenchymal stem cells regenerate skin tissue. Tissue Eng 11: 110-119. [PubMed]

3. Fu, L. Fang, X. Li, B. Cheng, Z. Sheng (2006) Enhanced wound-healing quality with bone marrow mesenchymal stem cells autografting after skin injury. Wound Repair Regen 14: 325-335. [PubMed]

4. Balaji S, Keswani SG, Crombleholme TM (2012) The Role of Mesenchyma Stem Cells in the Regenerative Wound Healing Phenotype. Adv Wound Care (New Rochelle) 1: 159-165. [PubMed]

5. Némos C, Basciano L, Dalloul A (2012) Biological effects and potential applications of mesenchymal stem cell culture under low oxygen pressure. Pathol. Biol. (Paris) 60: 193-198. [PubMed]

6. G. Ferrari, Cusella-De Angelis G, Coletta M, Paolucci E, Stornaiuolo A, et al (1998) Muscle regeneration by bone marrow-derived myogenic progenitors. Science 279: 1528-1530. [PubMed]

7. Xynos A, Corbella P, Belmonte N, Zini R, Manfredini R, et al. (2010) Bone marrow-derived hematopoietic cells undergo myogenic differentiation following a Pax-7 independent pathway. Stem Cells 28: 965-973.[PubMed]

8. Orlic D, Kajstura J, Chimenti S, Bodine DM, Leri A, et al. (2003) Bone marrow stem cells regenerate infarcted myocardium. Pediatr Transplant 7: 86-88. [PubMed]

9. Chen L, Wang DL, Wei ZR, Wang B, Qi JP, et al. (2016) Effects of local transplantation of autologous adipose-derived mesenchymal stem cells on the formation of hyperplastic scar on rabbit ears. Zhonghua Shao Shang Za Zhi 32: 582-587. [PubMed]

10. Lo Furno D, Mannino G, Cardile V, Parenti R, Giuffrida R (2016) Potential Therapeutic Applications of Adipose-Derived Mesenchymal Stem Cells. Stem Cells Dev. [PubMed]

11. Lee EY, Xia Y, Kim WS, Kim MH, Kim TH, et al. (2009) Hypoxia-enhanced wound-healing function of adipose-derived stem cells: increase in stem cell proliferation and up-regulation of VEGF and bFGF. Wound Repair Regen 17 : 540-547. [PubMed]

12. Jackson WM, Nesti LJ, Tuan RS (2012) Mesenchymal stem cell therapy for attenuation of scar formation during wound healing. Stem Cell Res Ther 3: 20 . [PubMed]

13. Cha J, Falanga V (2007) Stem cells in cutaneous wound healing. Clin. Dermato 25: 73-78. [PubMed]

14. Chen D, Hao H, Fu X, Han W (2016) Insight into Reepithelialization: How Do Mesenchymal Stem Cells Perform? Stem Cells Int pp: 6120173.

15. Cao PF, Xu YB, Tang JM, Yang RH, Liu XS (2014) HOXA9 regulates angiogenesis in human hypertrophic scars: Induction of VEGF secretion by epidermal stem cells. Int J Clin Exp Pathol 7: 2998-3007. [PubMed]

16. Coulombe PA (2003) Wound epithelialization: accelerating the pace of discovery. J Invest Dermatol 121: 219-230. [PubMed]

17. Wang H, Chen Z, Li XJ, Ma L, Tang YL (2015) Anti-inflammatory cytokine TSG6 inhibits hypertrophic scar formation in a rabbit ear model. Eur $\mathrm{J}$ Pharmacol 751: 42-49. [PubMed]

18. Waelti ER, Inaebnit SP, Hunziker T, Limat A, Braathen LR, et al. (1992) Coculture of human keratinocytes on post-mitotic human dermal fibroblast feeder cells: production of large amounts of interleukin 6. J Invest Dermatol 98: 805808 .

19. Smola H, Thiekötter G, Fusenig NE (1993) Mutual induction of growth factor gene expression by epidermal-dermal cell interaction. J Cell Biol 122: 417-429. [PubMed]

20. Maas-Szabowski N, Fusenig NE (1996) Interleukin-1 induced growthfactor expression in postmitotic and resting fibroblasts.J Invest Dermatol 107: 849855. [PubMed]

21. Grøn B, Stoltze K, Andersson A, Dabelsteen E (2002) Oral fibroblasts produce more HGF and KGF than skin fibroblasts in response to co-culture with keratinocytes. APMIS 110: 892-898. [PubMed] 
Citation: Dososaputro I, Hoekstra LT, Rahmania D, Perdanakusuma DS (2017) Role of Adipose-Derived Mesenchymal Stem Cells in Full Thickness Wound Healing. J Stem Cell Res Ther 7: 398. doi: 10.4172/2157-7633.1000398

Page 6 of 6

22. Zimmerlin L, Park TS, Zambidis ET, Donnenberg VS, Donnenberg AD Mesenchymal stem cell secretome and regenerative therapy after cancer. Biochimie 95: 2235-2245. [PubMed]

23. Frenette PS, Pinho S, Lucas D, Scheiermann C (2013) Mesenchymal stem cell: keystone of the hematopoietic stem cell niche and a stepping-stone for regenerative medicine. Annu Rev Immunol 31: 285-316. [PubMed]

24. Sugiyama H1, Maeda K, Yamato M, Hayashi R, Soma T, et al. (2008) Human adipose tissue-derived mesenchymal stem cells as a novel feeder layer for epithelial cells. J Tissue Eng Regen Med 2: 445-449. [PubMed]
25. Maharlooei MK, Bagheri M, Solhjou Z, Jahromi BM, Akrami M, et al. (2011) Adipose tissue derived mesenchymal stem cell (AD-MSC) promotes skin wound healing in diabetic rats. Diabetes Res Clin Pract 93: 228-234. [PubMed]

26. Du W, Li X, Chi Y, Ma F, Li Z, et al. (2016) VCAM-1+ placenta chorionic villiderived mesenchymal stem cells display potent pro-angiogenic activity. Stem Cell Res Ther 7: 49. [PubMed]

27. Czapla J, Matuszczak S, Wiśniewska E, Jarosz-Biej M, Smolarczyk R, et al. (2016) Human Cardiac Mesenchymal Stromal Cells with CD105+CD34Phenotype Enhance the Function of Post-Infarction Heart in Mice. PLoS One 11: 0158745. [PubMed] 